\section{Implications of Sarcopenia in Major Surgery}

\author{
Jeffrey Friedman, $\mathrm{MSE}^{1,2}$; Alisha Lussiez, $\mathrm{BSE}^{1,2}$; June Sullivan, $\mathrm{MBA}^{1,2}$; \\ Stewart Wang, MD, $\mathbf{P h D}^{1,2}$; and Michael Englesbe, $\mathbf{M D}^{1,2}$
}

Nutrition in Clinical Practice

Volume 30 Number 2

April 2015 175-179

(C) 2015 American Society

for Parenteral and Enteral Nutrition DOI: $10.1177 / 0884533615569888$

ncp.sagepub.com

hosted at

online.sagepub.com

(S)AGE

\begin{abstract}
Background: Sarcopenia, defined as a decrease in skeletal muscle mass and strength, is an important risk factor in clinical medicine associated with frailty, mortality, and worse surgical and nonsurgical outcomes. Conventional measures of sarcopenia rely on the subjective "eyeball test" and do not adequately describe risk. Computed tomography (CT) imaging studies may be used to objectively measure sarcopenia and may be used for surgical risk stratification and identification of patients for inclusion in a novel clinical remediation program. Methods: We describe results observed in the general, vascular, and liver transplant surgery populations determined by analytic morphomics - an analysis of CT scans in a semiautomated process using MATLAB v13.0. A perioperative optimization program has been implemented with the objective of remediating sarcopenia through improvement of patient mental and physical status prior to surgery. Results: Using analytic morphomics, we have noted significantly higher cost and increased rates of mortality and surgical complications among sarcopenic patients. The training program shows initial success, and among participating patients, we have observed reductions in payer and hospital costs and a decrease in length of hospital stay for patients following surgery. Conclusions: Through analytic morphomics, we are able to quantify markers of sarcopenia and identify patients at risk for increased mortality and poor surgical outcomes. Early identification of patients offers us the opportunity to remediate sarcopenia through perioperative training and support. Participating patients spend less time in the hospital and have lower healthcare costs. This program has the potential to improve the perioperative patient experience and ease financial burdens. (Nutr Clin Pract. 2015;30:175-179)
\end{abstract}

\title{
Keywords
}

surgery; sarcopenia; nutritional support; nutrition status; x-ray computed tomography; postoperative complications; risk assessment

Understanding patient risk factors for surgical complications and mortality allows patients, families, and clinicians to titrate the intensity of optimal care. Clinicians use several methods of risk assessment as they recommend a treatment plan. Most often, they identify risk using a subjective assessment of each patient's vigor and physiologic reserve. ${ }^{1,2}$ Developing techniques to objectively measure the same characteristics that form the gestalt of the "eyeball test" may provide improved means of risk stratification that can be more easily communicated to patients. $^{2}$ Understanding the patient factors and physiologic mechanisms underlying high-risk patients will elucidate targets to manage remediable risk and improve outcomes. The purpose of this article is to define sarcopenia, its clinical measurement using computed tomography (CT) scans, and the implication of those measurements in predicting surgical and mortality risk. We conclude this article with a brief discussion of proposed clinical implementation of risk factor remediation.

\section{Defining Sarcopenia and Its Clinical Relevance}

Sarcopenia, defined as the loss of skeletal muscle mass and strength, is associated with frailty, mortality, and poor outcomes in both surgical and nonsurgical patients. ${ }^{1,3-10}$ Patients with sarcopenia are particularly vulnerable in the setting of major physiologic stressors such as major surgery or surgical complications. The development of sarcopenia is multimodal; factors include malnutrition, functional status, and comorbid disease such as cancer, age, and heredity. Progressive resistance training and nutrition modifications are well-established interventions to reverse sarcopenia. ${ }^{3}$ Systematically identifying sarcopenic patients prior to major surgery and intervening on their potentially remediable risk factors may be a novel target for surgical quality improvement.

\footnotetext{
From the ${ }^{1}$ Department of Surgery, University of Michigan Health System, Ann Arbor, Michigan, and ${ }^{2}$ Morphomic Analysis Group, University of Michigan Health System, Ann Arbor, Michigan.
}

Financial disclosure: M.E., J.S., and S.W. are equity stakeholders in Prehab Technologies, LLC. This company was formed to deliver the technologies used as part of our prehabilitation program as part of our grant from Medicare, a CMS Innovation Grant that now supports implementation of MSHOP across the state of Michigan.

This article originally appeared online on February 13, 2015.

\section{Corresponding Author:}

Michael Englesbe, MD, University of Michigan Health System, 2926A

Taubman Center, 1500 East Medical Center Drive, Ann Arbor, MI 48109-5331, USA.

Email: englesbe@med.umich.edu 


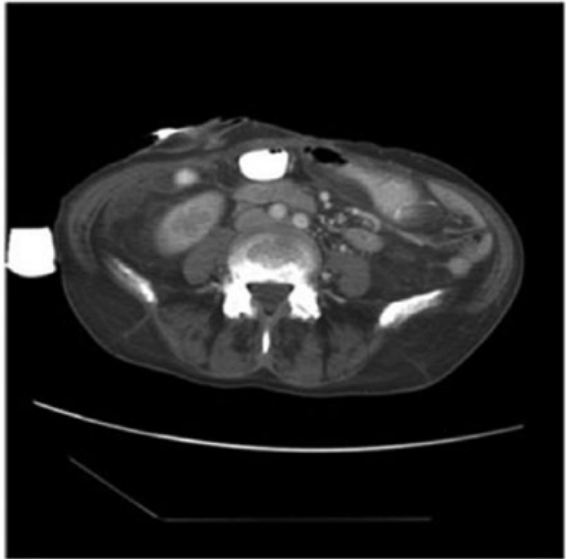

58 year old male diabetes, previous myocardial infarction, and COPD who is pre-operative for a colostomy takedown

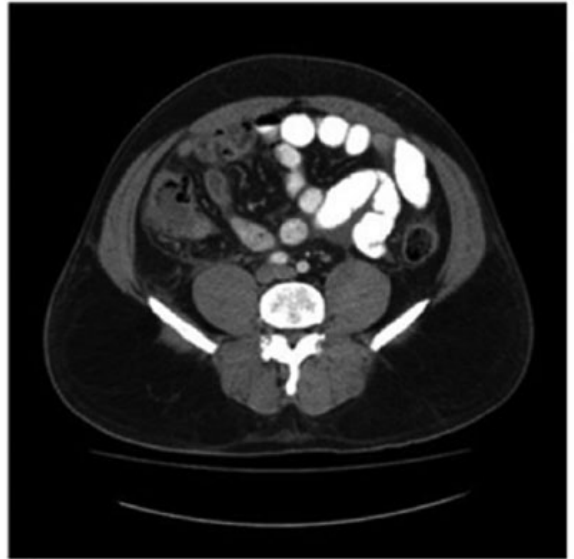

58 year old male diabetes, previous myocardial infarction, and COPD who is pre-operative for a colostomy takedown

Figure 1. Computed tomography scan comparison showing body composition differences between a sarcopenic (left) and nonsarcopenic (right) patient. Note the smaller and less clearly defined muscle groups adjacent to the vertebra of the sarcopenic patient compared with the robust and clearly defined muscle groups of the nonsarcopenic patient. COPD, chronic obstructive pulmonary disease. Adapted with permission from Englesbe MJ, Lee JS, He K, et al. Analytic morphomics, core muscle size, and surgical outcomes. Ann Surg. 2012;256(2):255-261.

\section{Using Preoperative Imaging for Risk Assessment}

Prior to major surgery, patients undergo an extensive radiographic workup. These images determine suitability for surgery and allow the surgeon to formulate an operative plan. Surgeons evaluate the areas of pathology, while the rest of the patient's body is not assessed. Radiographic imaging offers an untapped resource for risk stratification. Quantified global assessments of patient health and risk can be determined from these same preoperative imaging studies. ${ }^{2,4,6,7,9-11}$ An example can be seen in Figure 1, which displays cross-sectional CT images taken at the level of the fourth lumbar vertebrae of two 58-year-old male patients with a history of diabetes, chronic obstructive pulmonary disease (COPD), and myocardial infarction who are preparing for elective sigmoid colectomy. By conventional risk stratification and the "eyeball test," these patients may look similar. ${ }^{2}$ However, important differences are demarcated through quantified body composition measurements calculated using preoperative CT imaging studies, a process called analytic morphomics.

\section{Investigating the Utility of Analytic Morphomics}

Beyond the obvious visual disparity observed in patient scans, quantitative measures of body composition can be made to determine objective measures of surgical risk.
Analytic morphomic processing is completed on CT images in a semiautomated manner using algorithms programmed in MATLAB v13.0 (MathWorks, Natick, MA). Our work notes that sarcopenia within the trunk muscles is a dominant risk factor for poor outcomes. The psoas muscles, the paraspinous muscles, and the thigh muscles offer reliable measures of sarcopenia in CT cross-sectional imaging. ${ }^{2,4,6,7,11}$ As shown in Figure 2, an examination of the psoas muscles at the fourth lumbar vertebrae in major, elective general and vascular surgery patients revealed that trunk muscle area is normally distributed, and extremes of the population are notably different. Examination of the upper and lower tertiles of core muscle size (controlling for relevant patient risk factors and operative case complexity) shows 1-year survival to be $95 \%$ vs $87 \%$ and 3 -year survival to be $91 \%$ vs $75 \%$, respectively. ${ }^{9}$ Among patients with chronic liver disease undergoing liver transplantation, the association of core muscle size with survival is even more dramatic. Upper quartile patients experience $87 \%$ one-year survival and $77 \%$ three-year survival vs $50 \%$ and $26 \%$ survival, respectively, among lower quartile patients. ${ }^{4}$ Importantly, core muscle size is the strongest predictor of survival. In addition to mortality, sarcopenia is associated with surgical complications. ${ }^{12}$ Among general and vascular surgery patients, average risk of complication is $12 \%, 15 \%$, and $21 \%$, respectively for the upper, middle, and lower tertiles of core muscle size. ${ }^{9}$

Other morphomic measures of body composition, such as subcutaneous fat, correlate with surgical outcomes. Among 


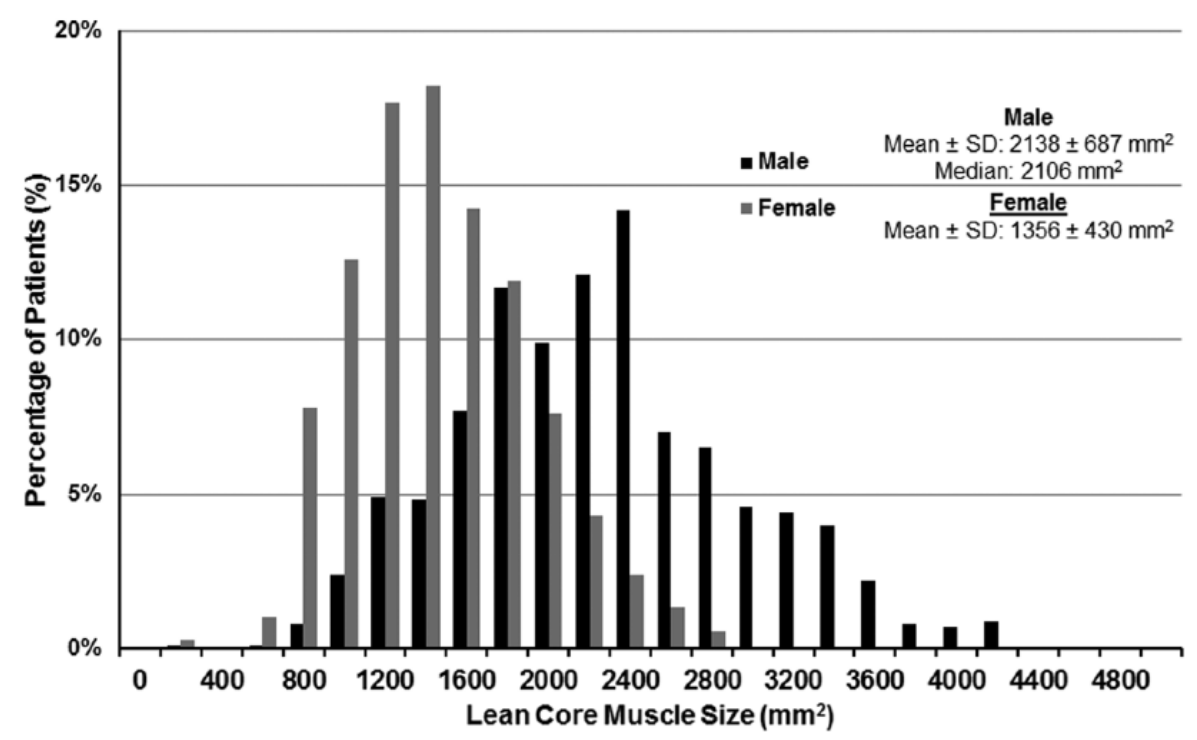

Figure 2. Distribution of lean psoas muscle area in males and females. Adapted with permission from Englesbe MJ, Lee JS, He K, et al. Analytic morphomics, core muscle size, and surgical outcomes. Ann Surg. 2012;256(2):255-261.

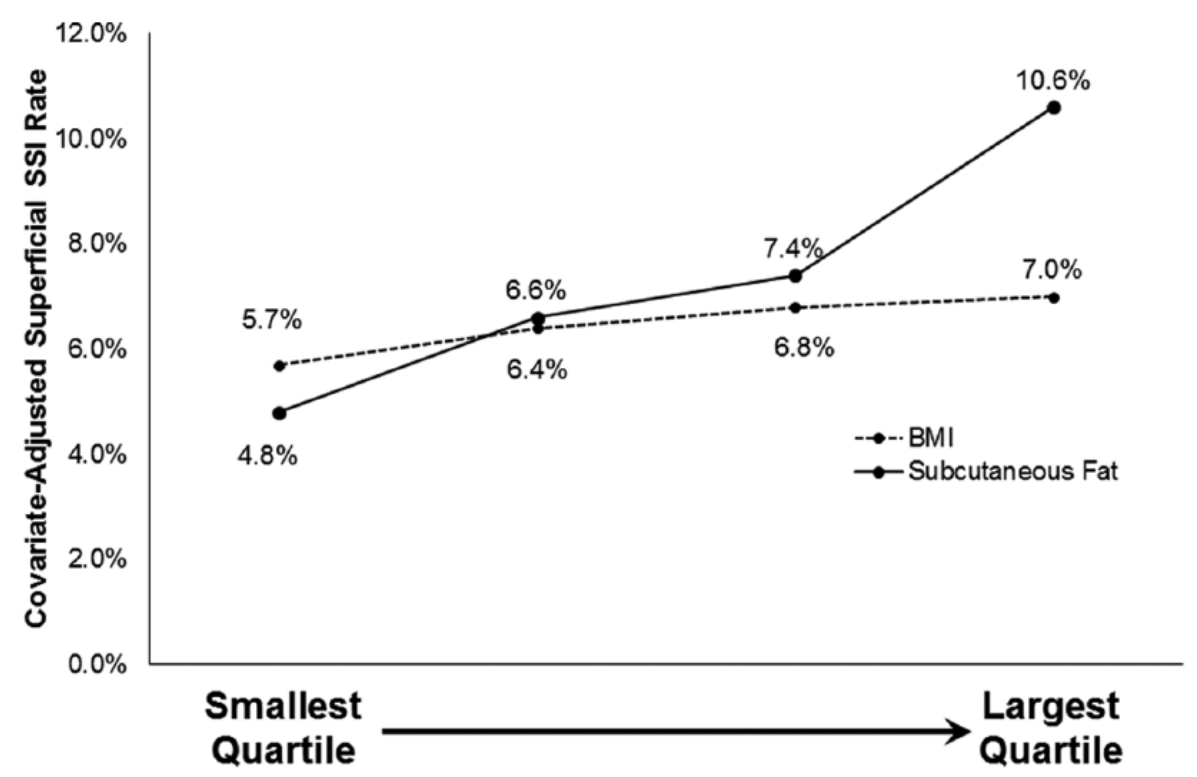

Figure 3. Comparison of surgical site infection rates by body mass index (BMI) and subcutaneous fat measurement.

patients undergoing a midline laparotomy, increased subcutaneous fat is a predictor of superficial, incisional surgical site infection (SSI). ${ }^{10}$ Figure 3 displays rate of SSI by quartile of body mass index (BMI) and subcutaneous fat after adjusting for other independent predictors, including tobacco use, steroids, American Society of Anesthesiologists (ASA) risk class, and operative time. Importantly, subcutaneous fat measurement better discriminates risk compared with BMI.
Patients with sarcopenia are significantly more expensive to care for. For patients undergoing major general or vascular surgery, decreasing lean core muscle size is associated with increasing costs for insurers. Sarcopenic patients had a mean payer cost of $\$ 34,796$, while nonsarcopenic patients had a mean payer cost of $\$ 21,380 .^{6}$ Figure 4 displays this relationship by percentile of lean psoas area and payer costs for major elective abdominal surgery. 


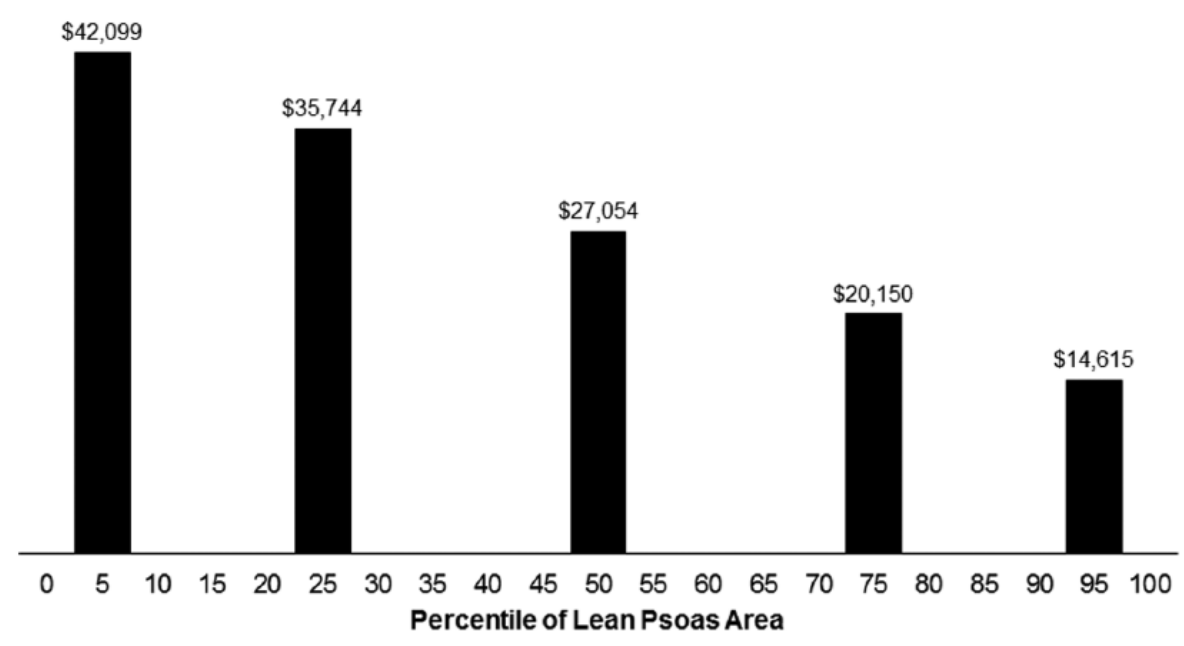

Figure 4. Mean adjusted payer cost for major general elective surgery by lean psoas area. Costs describe expenses incurred over the period of 3 days prior to surgery through postoperative discharge.

\section{Sarcopenia: What Can Be Done Prior to Surgery?}

Understanding the financial implications of sarcopenia is critical to drive change. Interventions such as preoperative physical training and nutrition intervention are expensive. Furthermore, the costs of these interventions are not easily recoverable, since they lie outside of the surgical episode payments. Most clinicians are easily convinced that presurgical interventions focused on sarcopenic and malnourished patients are good for patient care, making developing programs that are financially viable a must.

Investment in programs to optimize patients prior to surgery is good business for hospitals and payers. Of course, giving the best care possible is a priority for all stakeholders, and patient satisfaction is good for business. Beyond this, targeted preoperative interventions save money. This is rooted in the observations detailed above; sarcopenic patients are high risk and expensive.

Exercise training can rapidly and significantly improve functional health, especially in deconditioned individuals. ${ }^{13-16}$ Preoperative exercise may improve surgical outcomes and is feasible, although it has not been accepted as standard of care and is not normal clinical practice. ${ }^{17-20}$ Nutrition assessment and intervention are also key components of these interventions and must be done efficiently so as not to add unnecessary costs.

\section{Implementing a Program for Remediation of Perioperative Risk}

Motivated by our work in sarcopenia, we began studying and implementing perioperative optimization as the standard of care for all patients. Much as an athlete trains for competition, patients should train for surgery by reducing remediable risk, including sarcopenia. The Michigan Surgical Home \& Optimization Program (MSHOP) is a preoperative training program that includes physical exercise, cessation of smoking, stress reduction, nutrition support, and daily spirometer exercises that is being implemented at the University of Michigan. More than 350 patients have been enrolled in the pilot program.

The primary outcomes for this clinical program have been related to the patient-centered experience and financial data. Feedback from families and patients has been uniformly positive. Patients feel engaged with the perioperative care process and in control of their outcomes. To assess the financial outcomes of the program, we matched participating patients to historical controls based on age, sex, ASA risk class, case complexity, and Medicare Allowable Payments using standard propensity matching techniques. The preliminary results note a consistent reduction in costs for the payer and hospital of and a 2-day reduction in length of stay in the hospital following surgery.

The genesis of MSHOP was the observation that patients with small muscles on preoperative imaging do poorly with major surgery. Early trials focused on muscle strength interventions. It soon became clear that the severity of illness and complexity of the patient's nutrition status made rigorous assessments of sarcopenia interventions challenging. We eventually moved toward a commonsense clinical program, focusing on a broad range of interventions. Which interventions are most effective is not yet known, but all are low cost with the exception of aggressive nutrition modifications for malnourished patients. Most important, MSHOP has facilitated a cultural change. Our expectation is that all patients train for elective surgery. In our experience, most patients embrace this opportunity. The program empowers patients 
during a time of great fear and uncertainty. As we use novel techniques such as CT scans to assess patient-specific risk factors for poor outcomes, clinical programs to mitigate remediable risk will become tailored for each patient.

\section{Conclusion}

Use of analytic morphomics can aid in patient risk stratification. Identifying sarcopenic patients and correcting their remediable risk factors prior to surgery is of the utmost importance since this patient cohort has worse surgical outcomes, increased mortality rates, and higher total payer costs compared with patients without sarcopenia. We recommend the use of a preoperative training program for patients with remediable perioperative risk factors to help improve outcomes, lower costs, and decrease length of stay.

\section{References}

1. Dodds R, Sayer AA. Sarcopenia. Arq Bras Endocrinol Metab. 2014;58(5):464-469.

2. Englesbe MJ. Quantifying the eyeball test: sarcopenia, analytic morphomics, and liver transplantation. Liver Transplant. 2012;18(10):1136-1137.

3. Cruz-Jentoft AJ, Landi F, Schneider SM, Zúñiga C, et al. Prevalence of and interventions for sarcopenia in ageing adults: a systematic review. Age Ageing. 2014;43(6):748-759.

4. Englesbe MJ, Patel SP, He K, et al. Sarcopenia and mortality after liver transplantation. J Am Coll Surg. 2010;211:271-278.

5. Tan BH, Birdsell LA, Martin L, et al. Sarcopenia in an overweight or obese patient is an adverse prognostic factor in pancreatic cancer. Clin Cancer Res. 2009;15:6973-6979.

6. Sheetz KH, Waits SA, Terjimanian MN, et al. Cost of major surgery in the sarcopenic patient. J Am Coll Surg. 2013;217(5):813-818.

7. Lee JS, He K, Harbaugh CM, et al. Frailty, core muscle size, and mortality in patients undergoing open abdominal aortic aneurysm repair. J Vasc Surg. 2011;53:912-917.
8. Lieffers JR, Bathe OF, Fassbender K, et al. Sarcopenia is associated with postoperative infection and delayed recovery from colorectal cancer resection surgery. Br J Cancer. 2012;107:931-936.

9. Englesbe MJ, Lee JS, He K, et al. Analytic morphomics, core muscle size, and surgical outcomes. Ann Surg. 2012;256:255-261.

10. Lee JS, Terjimanian MN, Tishberg LM, et al. Surgical site infection and analytic morphometric assessment of body composition in patients undergoing midline laparotomy. J Am Coll Surg. 2011;213(2): 236-244.

11. Mazurek AA, Canvasser LD, Cron DC, et al. Paraspinous muscle as a predictor of surgical outcome. J Surg Res. 2014;186(2):497.

12. Peng PD, Van vledder MG, Tsai S, et al. Sarcopenia negatively impacts short-term outcomes in patients undergoing hepatic resection for colorectal liver metastasis. HPB (Oxford). 2011;13(7):439-446.

13. Kujala UM. Is it really important for sick people to perform exercise? Scand J Med Sci Sports. 2006;16(3):145-146.

14. Kujala UM. Benefits of exercise therapy for chronic diseases. Br J Sports Med. 2006;40(1):3-4.

15. Kujala UM. Evidence on the effects of exercise therapy in the treatment of chronic disease. Br J Sports Med. 2009;43(8):550-555.

16. Kujala UM. Evidence for exercise therapy in the treatment of chronic disease based on at least three randomized controlled trials - summary of published systematic reviews. Scand J Med Sci Sports. 2004;14(6): 339-345.

17. Valkenet K, van de Port IG, Dronkers JJ, et al. The effects of preoperative exercise therapy on postoperative outcome: a systematic review. Clin Rehabil. 2011;25(2):99-111.

18. Carli F, Zavorsky GS. Optimizing functional exercise capacity in the elderly surgical population. Curr Opin Clin Nutr Metab Care. 2005;8(1):23-32.

19. Dronkers JJ, Lamberts H, Reutelingsperger IM, et al. Preoperative therapeutic programme for elderly patients scheduled for elective abdominal oncological surgery: a randomized controlled pilot study. Clin Rehabil. 2010;24(7):614-622.

20. Hoogeboom TJ, Dronkers JJ, van den Ende CH, et al. Preoperative therapeutic exercise in frail elderly scheduled for total hip replacement: a randomized pilot trial. Clin Rehabil. 2010;24(10):901-910. 